\title{
Abnormal Femoral Origin of the Anterior Cruciate Ligament Associated with Osteochondritis Dissecans of the Medial Femoral Condyle*
}

\author{
Massimiliano Salvi", Francesco Caputo, Giuseppe Piu, Marco Sanna, Cristina Sanna \\ Department of Prosthetic and Sports Surgery, Sant’Elena Private Hospital, Kinetika Group, Quartu Sant'Elena, Cagliari, Italy \\ Email: "massimiliano.salvi@tin.it
}

Received September 27, 2013; revised October 20, 2013; accepted October 27, 2013

Copyright (C) 2013 Massimiliano Salvi et al. This is an open access article distributed under the Creative Commons Attribution License, which permits unrestricted use, distribution, and reproduction in any medium, provided the original work is properly cited.

\begin{abstract}
We report a case of abnormal meniscal-like anterior cruciate ligament (ACL), inserting at the 9 o'clock position just beside the cartilage of the lateral femoral condyle, in a stable knee associated with osteochondritis dissecans (OCD) of the medial femoral condyle.
\end{abstract}

Keywords: Congenital Anomaly; Abnormal Anterior Cruciate Ligament; Femoral Attachment; Osteochondritis Dissecans

\section{Introduction}

Abnormal femoral or tibial attachments of the ACL are very rare. To our knowledge, there have been only two reported cases of abnormal femoral origin of the ACL, one combined with a discoid lateral meniscus [1] and one isolated [2]; two reported cases of abnormal tibial attachment of the ACL [3] and one reported case of OCD of the medial femoral condyle associated with ACL and lateral meniscus hypoplasia [4].

We report a case of abnormal femoral origin of the ACL associated with OCD of the medial femoral condyle.

\section{Case Report}

A 17-year-old male presented to our clinic with one-year history of swelling, pain and locking in his right knee, during daily activities and mild recreational sports activity, with no history of trauma. The patient was $170 \mathrm{~cm}$ tall and weighed $58 \mathrm{Kg}$.

The left knee was asymptomatic. The right knee was swollen, and two large loose bodies were palpable at the suprapatellar pouch. The knee was locked in $15^{\circ}$ of flexion, and the active range of motion was from $15^{\circ}$ to full flexion.

Clinical lower limb alignment was normal. Both knees

${ }^{*}$ Conflict of interest: The Authors declare that they have no conflict of interest.

${ }^{\#}$ Corresponding author. were stable in anterior and posterior drawer tests, varus and valgus stress tests, Lachman test and pivot shift test.

Plain radiographs revealed OCD of the medial femoral condyle at the classical site and two loose bodies. Magnetic resonance imaging showed apparent normal cruciate ligaments, normal menisci and cartilage of the lateral and patello-femoral compartments, a large OCD of the medial femoral condyle and three loose bodies (Figure 1).

The patient was scheduled for arthroscopic evaluation and treatment of OCD and loose bodies removal.

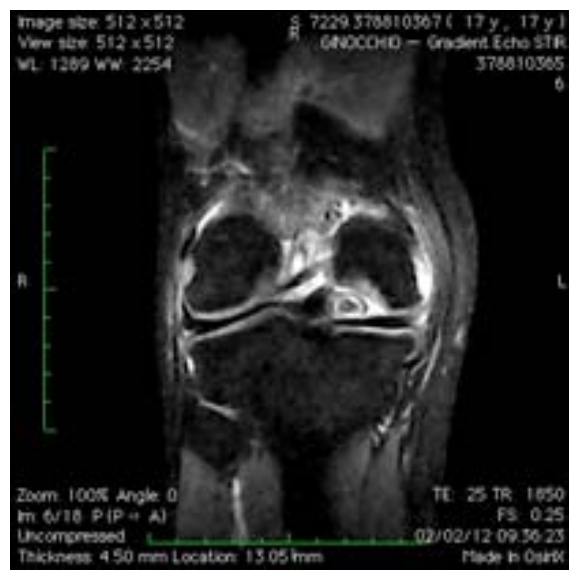

Figure 1. Gradient echo STIR magnetic resonance image showing a large osteochondritis dissecans of the medial femoral condyle and a loose body. 
Arthroscopy was performed using the standard anterolateral and anteromedial portals and revealed no meniscus anomalies or tears and no cartilage damage of the lateral femoral condyle, sulcus and patellae.

A large $(2 \times 4 \mathrm{~cm})$ OCD of the medial femoral condyle and five loose bodies, two of which were locatedin the posteromedial compartment, were found.

The morphology of the ACL was surprisingly clearly abnormal. A flat meniscal-like ACL, inserting just beside the cartilage of the lateral femoral condyle at the 9 o'clock position over the whole length of the shallowdeep direction was found (Figures 2(a)-(c)). The tibial attachment of the ACL appeared to be normal.

Loose bodies were removed through the standard portals and a posteromedial portal, and the OCD was treated by microfractures. The abnormal femoral insertion of the ACL was left untreated. The patient was recommended to abstain from weight bearing activity and use two crutches for six weeks.

Six months postoperatively, the patient had no pain, swelling or limitation to the range of motion, and here turned to his normal lifestyle.

\section{Discussion}

OCD of the medial or lateral femoral condyles combined with malformation of the menisci $[5,6]$ or associated with congenital hypoplasia of the ACL and lateral meniscus

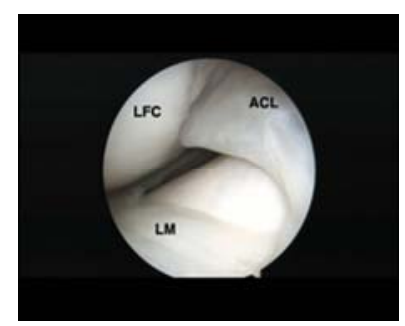

(a)

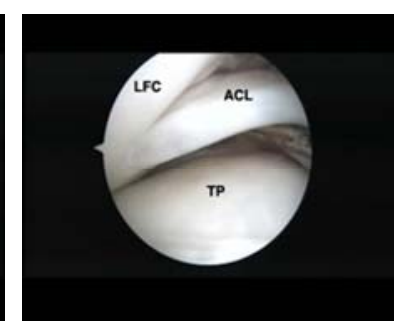

(b)

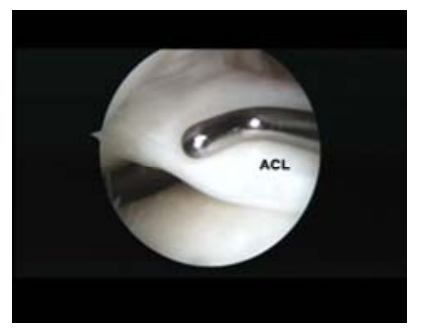

(c)

Figure 2. (a), (b) Arthroscopic view of the right knee from a standard anterolateral portal, and a meniscal-shaped abnormal attachment of the anterior cruciate ligament, just beside the cartilage of the lateral femoral condyle. (c) The anterior cruciate ligament inserts over the whole length of the intercondylar notch in a shallow-deep direction at the 9 o'clock position. ACL: Anterior Cruciate Ligament; LFC: Lateral Femoral Condyle; TP: Tibial Plateau; LM: Lateral Meniscus.
[4] have been previously reported in the literature.

However, abnormal femoral or tibial attachments of the ACL are very rare.

Calpur et al. in 2004 [3] described two cases of unreported abnormally wide triangular-shaped tibial insertion of the ACL, causing painful anterior notch impingement.

To our knowledge, abnormal femoral origin of the ACL has been previously reported only in two cases, although neither of them was associated with OCD.

The first case was reported by Hoffmann in 1997 [1]. He found an abnormal femoral origin of the ALC, which is very similar to that of described in our case, and combined with a complete discoid lateral meniscus in a 3year-old child.

In that case, the femoral insertion was found very anterior in the intercondylar notch at the 10 o'clock position, just beside the articular cartilage of the lateral femoral condyle. Although the anomaly was completely asymptomatic, after the discoid lateral meniscus was resected into a normal-shaped lateral meniscus, the anterior part of the femoral insertion of the ACL was also resected, without causing instability at one-year followup.

In our case, the femoral insertion of the ACL was more horizontal, at a 9 o'clock position, compared with that described by Hoffmann. However, we did not resect the anterior portion of the ACL.

The second case was reported by Sarsilmaz and Gelal in 2011 [2].

They found an isolated congenital anomaly of the femoral insertion of the ACL in which the posterolateral bundle attached separately to an intraarticular large accessory ossicle, causing pain but not instability.

To our knowledge, this is the first reported congenital abnormal femoral origin of the ACL associated with OCD of the medial femoral condyle.

However, because this type of abnormal femoral attachment of the ACL does not compromise ligament function, we believe that it should not be treated.

\section{REFERENCES}

[1] F. F. Hoffmann, "Abnormal Femoral Origin of the Anterior Cruciate Ligament Combined with a Discoid Lateral Meniscus,” Arthroscopy, Vol. 13, No. 2, 1997, pp. 254256. http://dx.doi.org/10.1016/S0749-8063(97)90165-1

[2] A. Sarsilmaz and F. Gelal, "A New Variation: An Anterior Cruciate Ligament Attached to the Accessory Ossicle,” Clinical Anatomy, Vol. 24, No. 8, 2011, pp. 991-993. http://dx.doi.org/10.1002/ca.21113

[3] O. U. Calpur, M. Ozcan and H. Gurbuz, "Deltoid (Triangular)-Shaped Anterior Cruciate Ligament that Caused Notch Impingement: A Report of Two Cases," Arthroscopy, Vol. 20, No. 6, 2004, pp. 637-640. http://dx.doi.org/10.1016/j.arthro.2004.04.061 
[4] T. Mitsuoka, S. Horibe and M. Hamada, “Osteochondritis Dissecans of the Medial Femoral Condyle Associated with Congenital Hypoplasia of the Lateral Meniscus and Anterior Cruciate Ligament,” Arthroscopy, Vol. 14, No. 6, 1998, pp. 630-633.

http://dx.doi.org/10.1016/S0749-8063(98)70063-5

[5] P. M. Aichroth, D. V. Patel and C. L. Marx, "Congenital Discoid Lateral Meniscus in Children. A Follow-Up Study and Evolution of Management," Journal of Bone and Joint Surgery (British Volume), Vol. 73, No. 6, 1991, pp. 932-936.

[6] T. Beyzadeoglu, A. Gokce and H. Bekler, "Osteochondritis Dissecans of the Medial Femoral Condyle Associated with Malformation of the Menisci," Orthopedics, Vol. 31, No. 5, 2008, p. 504.

http://dx.doi.org/10.3928/01477447-20080501-02 